\title{
Decrease of hot regions in the polar corona of the Sun during 1957-2002
}

\author{
D. K. Callebaut ${ }^{1}$, A. G. Tlatov ${ }^{2}$ and V. I. Makarov ${ }^{2}$ \\ ${ }^{1}$ Physics Department, CGB, University of Antwerp, B-2020 Antwerp, Belgium \\ email: Dirk.Callebaut@ua.ac.be \\ ${ }^{2}$ Main (Pulkovo) Astronomical Observatory, St. Petersburg 196140, Russia \\ email: Solar@narzan.com, makarov@gao.spb.ru
}

\begin{abstract}
The Kislovodsk series of the green (FeXIV $\left.530.3 \mathrm{~nm} ; \mathrm{KI}_{5303}\right)$ and red (FeX $637.4 \mathrm{~nm}$; $\left.\mathrm{KI}_{6374}\right)$ corona has been used to calculate the mean monthly intensities at high latitude $\left(45^{\circ}-\right.$ $90^{\circ}$ ) zones for $1957-2002$. The ratio $\mathrm{KI}_{6374} / \mathrm{KI}_{5303}$ was observed during the minimum sunspot activity. It was shown that this ratio increased by more than a factor 2 during the last 45 years at the high latitudes $\left(45^{\circ}-90^{\circ}\right)$. This may be interpreted that the fraction of cool regions in the polar corona has more than doubled over these years. We suggest that this increase in the amount of cool regions is related to the increase in the area of the polar zones occupied by magnetic field of a single polarity at the solar minimum and possibly to a corresponding increase in the area occupied by polar coronal holes, while the magnetic field strength itself has not or barely increased.
\end{abstract}

Keywords. Sun: activity, corona, filaments, magnetic fields

\section{Introduction}

The most conspicuous phenomena on the Sun are obviously the sunspots and their features, especially their butterfly diagram, are well known, although their generating mechanism is barely at the beginning of being understood, Callebaut (2006). Next main phenomenon on the solar surface are the polar faculae. As their name indicates they appear above say $40^{\circ}$. They have kilogauss fields according to Homann, Kneer, \& Makarov (1998), their individual area is an order of magnitude smaller than the spots, their lifetime (minutes to days) is much shorter than for spots (up to months), but they outnumber the spots by at least an order of magnitude, so that their total flux is somewhat larger than the one of the spots. The polar faculae have a butterfly diagram too and this shows a Schwabe cycle too (and probably the Hale cycle as well). Moreover some of their features (their peaks) precede the spots by about 5.6 years (about half a Schwabe cycle) although their total number does not show a direct relation to the Wolf number, Makarov, Makarova, \& Callebaut (2006).

The large-scale unipolar magnetic field regions (global field regions in short) constitute the third main phenomenon on the solar surface. These are often neglected as their fields are only a few gauss, see Callebaut, \& Makarov (1992), but they cover the whole surface of the Sun and their total flux may exceed the one by the polar faculae. Their number varies between 4 and 8 , in half of them the field dives in the Sun, in the other half the field points out of the Sun. They are revealed by $H_{\alpha}$ synoptic charts since 1878 . At their boundaries appear filament bands (filaments and filament channels) surrounding the whole Sun. These boundaries move toward the poles and when one of them reaches a pole this causes a polar reversal, after which the next boundaries (in the same hemisphere) jump somewhat back to a lower latitude, where they stay for about a year during solar 
minimum, oscillating a few degrees around what we call their 'rest-latitude'. When a new cycle begins new global field regions are generated near the equator and the existing ones resume their motion to the poles, Callebaut, Makarov \& Tlatov (2003). Now the upper 'rest-latitudes' were situated at $53^{\circ}$ (average north and south) in 1878 (cycle 12) and came down to $38^{\circ}$ at cycle 23 . The lower 'rest-latitudes' started some $22^{\circ}$ lower in cycle 12 and decreased fairly parallel to the upper ones. The decrease of the (higher) rest-latitudes resulted in practically a doubling of the polar cap, which may explain (see discussion below) the roughly doubling of the solar flux on the Earth as measured by the geomagnetic index. Moreover this was used, Makarov, Tlatov, Callebaut, et al. (2002), to show that the temperature on Earth had increased by about $1 \mathrm{~K}$ since the Maunder Minimum.

It seems plausible to consider the coronal holes as the key between the doubling of the polar cap and of the flux. However, the coronal holes are responsible for a major part of the escape of heat and the question arises whether or not the temperature in the corona has decreased during the last 50 years. This may be investigated by studying the evolution of the intensities of the green (FeXIV $530.3 \mathrm{~nm} ; \mathrm{KI}_{5303}$ ) and red (FeX $637.4 \mathrm{~nm} ; \mathrm{KI}_{6374}$ ) coronal lines. The ratio $\mathrm{KI}_{6374} / \mathrm{KI}_{5303}$ is used to estimate the coronal temperature and its evolution over the last half century. This paper continues the work of Makarov, Tlatov, \& Callebaut (2003) and Makarov, Tlatov, \& Callebaut (2006).

\section{Observational data}

At present there are 5 series of coronal intensity: Gnevyshev (1984); Sýkora (1992); Altrock (1998); Sakurai, Irie, Imai, et al. (1999); Tlatov, Guseva, \& Kim (2001); Makarov, \& Tlatov (2001); Rušin, \& Rybanský (2002). In fact there is no generally accepted long-term series of coronal intensities of the green and red lines. However, Makarov, Tlatov, \& Callebaut (2003) obtained by combination from the data mentioned above a fair long-term series of those coronal intensities: this was essentially by supplementing the Kislovodsk data by the other data. It may be noted that the Kislovodsk data are already a fair basis in themselves in view of several reasons: high stability during more than 50 years (same instruments, same procedure) and very regular observations. This reveals itself as well in the very good correspondence with the sunspot area or equivalently Wolf number, $W$ : calculating the cross-correlation functions for e.g. the FeXIV $503.3 \mathrm{~nm}$ intensity and $W$ gave

$\mathrm{IC}_{K I}=0.228 W+12.8$ (uci) with $r=0.91(1952-2002)$.

Here $r$ is the correlation factor and uci stands for 'unit of coronal intensity', Makarov, Tlatov, \& Callebaut (2006). The relation with other long-term changes of the polar activity of the Sun was discussed by Makarov, Tlatov, \& Callebaut (2005).

\section{Discussion}

\subsection{Coronal temperature}

The ratio $\mathrm{KI}_{6374} / \mathrm{KI}_{5303}$ was plotted in Makarov, Tlatov \& Callebaut (2003), Fig. 10, for the latitudes $45^{\circ}-90^{\circ}$ and Fig. 11 , for the latitudes $0^{\circ}-90^{\circ}$. The first figure reveals a correspondence with the Schwabe and Hale cycle, while the second shows a correspondence with the Schwabe cycle only. This is a very surprising feature which may be interpreted that the coronal holes in the sunspot region behave differently than those in the polar regions. We suggest that this is due to the fact that the polar regions have mainly one global magnetic field region while in the sunspot regions several global field regions occur, 
in particular it is there that new global field regions are generated. This means that in the equatorial region there is an accumulation of 3 global field regions (in each hemisphere), corresponding to 3 subsequent cycles, while this is not so for the polar region; hence the latter may more neatly show a relation with one Hale cycle.

The ratio $\mathrm{KI}_{6374} / \mathrm{KI}_{5303}$ for the latitudes $45^{\circ}-90^{\circ}$ varies between 0.15 to 0.5 for the latitudes $45^{\circ}-90^{\circ}$. It shows a faint long term increase. From the expression given by Guhathakurta, Fisher \& Altrock (1993) one may deduce that the temperature in the corona at level 1.04 changed from $1.5210^{6} \mathrm{~K}$ to $1.4210^{6} \mathrm{~K}$ (improving the values given in Makarov, Tlatov, \& Callebaut (2003)). The decrease is thus $0.110^{6} \mathrm{~K}$, which is relatively very small. Moreover, the fluctuations are around $0.0510^{6} \mathrm{~K}$.

\subsection{Flux versus field strength}

In fact the increase of the polar cap by the lowering of the upper rest-latitudes (see introduction) allows for more coronal holes in this polar cap and subsequently for a larger total magnetic flux (as manifested by the geomagnetic index). However, these effects may be fairly well proportional if the magnetic field itself is not changed. Then it seems plausible that the coronal temperature should not change. However, we can think of a reason why the magnetic field may increase: eventually the polar cap has expanded during the last half century at the expense of the equatorial (sunspot) region where the differential rotation is stronger; however, we expect that it is precisely a weakening of this differential rotation which allows the polar cap to expand at the expense of the sunspot region. In view of that we expect a rather small increase in magnetic field, although the flux is doubled as a consequence of the doubling of the surface of the polar cap. And this small increase in strength of the coronal holes might yield a small decrease in temperature of the corona as is somewhat suggested by the present analysis.

It may be noted that according to the results of Stamper, Lockwood, \& Wild (1999) the magnetic flux as indicated by the geomagnetic index may have slightly more than doubled in nearly a century and a half. However, Svalgaard, Cliver, \& Lesage (2003), showed that the value of the geomagnetic index was slightly larger in the 19th century than what was used by Stamper, Lockwood, \& Wild (1999), so that the solar magnetic field near the Earth did barely change. Moreover, according to figure 4 of Svalgaard, Cliver, \& Lesage (2003) the interplanetary magnetic field near the Earth from the $u$ measure 1872-1889 and from the IDV index 1890-2003 fluctuated practically around the same value although with some goodwill one can see a small average increase in the 20th century.

\section{Conclusions}

1) We discussed the decrease of about $0.110^{6} \mathrm{~K}$ in the corona obtained recently by us, Makarov, Tlatov, \& Callebaut (2003) and Makarov, Tlatov, \& Callebaut (2006). The doubling of the polar cap may be held responsible for the doubling of the magnetic flux near the Earth. However, the magnetic field itself near the Earth seems barely to have increased and it is doubtful whether this may have affected the temperature of the corona: the error flags are too large. This seems consistent with some theoretical arguments which suggest that the magnetic field in the polar cap should not increase or at most increase very little.

2) As the variation of the coronal temperature is in a subtle way related to the main phenomena on the solar surface (spots, polar faculae, global magnetic field regions) and solar atmosphere (heating and coronal holes) as well as to the magnetic flux reaching the 
Earth and the temperature and climate on the Earth, it seems important to investigate this temperature variation and its connections profoundly.

3) In the analyses in Makarov, Tlatov, \& Callebaut (2003) and Makarov, Tlatov, \& Callebaut (2006) we had divided rather arbitrarily the Equatorial and polar region at $45^{\circ}$. It seems suitable to repeat the analysis, however, taking the division according to the higher rest-latitude of the boundary of the global magnetic field regions. That means that the division is changing each cycle. This will throw more light on the observation that the polar region follows the Schwabe cycle and the Hale cycle, while the equatorial regions shows the Schwabe cycle.

4) We made use of the hypothesis that the ion and electron temperatures are the same. Actually the heating process of the corona is not yet well understood, while it is well known from plasma physics that this may affect strongly the temperature of each. A measurement to test this hypothesis of equal temperatures would be very welcome. Maybe the present analysis can be repeated with another coronal line: this third line may be combined once with the red line and once with the green one; comparison of the results would be revealing. This third line has not to be observed during the whole span of 50 years: even one year should be informative, although a full cycle should be better.

\section{References}

Altrock, R.C. 1998, in: R.S. Balasubramanian, J.W. Harvey and D.M. Rabin (eds.), Synoptic Solar Physics, APS Conference Series 140, 339

Badalyan, O.G., Obridko, V.N. \& Sýkora, J. 2001, Solar Phys. 199, 421

Callebaut, D.K. 2006, ApJ To be published

Callebaut, D.K., Makarov, V.I. \& Tlatov, A.G. 2003, in: 'Solar Variability as an Input to the Earth's Environnement', Proc. ISCS 2003 Symposium, TatranskaLomnica, Slovakia, 23-28 June 2003 (ESA SP-535, Sept. 2003), 749

Callebaut, D.K. \& Makarov, V.I. 1992, Solar Phys. 141, 381

Guhathakurta, M., Fisher, R.R. \& Altrock, R.C. 1993, ApJ L145, 414

Gnevyshev, M.N. 1984, Solar Phys. 90, 177

Homann, T.. Kneer, F. \& Makarov, V.I. 1998, Solar Phys. 175, 81

Makarov, V.I. \& Tlatov, A.G. 2001, in: V.I. Makarov, V. N. Obridko, (eds.), 'The Sun in Epoch of Magnetic Field Reversal', Proc of V Pulkovo International Conference on Solar Physics, St. Petersburg, Russia. 5, 259

Makarov, V.I., Makarova, V.V., \& Callebaut, D.K. 2006b, Solar Phys. in press

Makarov, V.I., Tlatov, A.G., Callebaut, D.K. \& Obridko, V.N. 2002, Solar Phys. 206, 383

Makarov, V.I., Tlatov, A.G. \& Callebaut, D.K. 2003, in: 'Solar Variability as an Input to the Earth's Environnement', Proc. ISCS 2003 Symposium, TatranskaLomnica, Slovakia, 23-28 June 2003 (ESA SP-535, Sept. 2003), 217

Makarov, V.I., Tlatov, A.G. \& Callebaut, D.K. 2005, Solar Phys. 224, 49

Makarov, V.I., Tlatov, A.G. \& Callebaut, D.K. 2006a, Solar Phys. in press

Rušin, V. \& Rybanský, M. 2002, Solar Phys. 207, 47

Sakurai, T., Irie, M., Imai, M., Miyazaki, H. \& Sýkora, J. 1999, Publ. Natl. Astron. Obs. Japan 5,121

Stamper, R., Lockwood, M., \& Wild, M.N. 1999, J. Geophys. Res. 104(A12), 24325

Svalgaard, L., Cliver, E. W., \& Lesage, P. 2003, in: 'Solar Variability as an Input to the Earth's Environnement', Proc. ISCS 2003 Symposium, TatranskaLomnica, Slovakia, 23-28 June 2003 (ESA SP-535, Sept. 2003), 15

Sýkora, J. 1992, Contrib. Astron. Obs. Skanate Pleso 22, 55

Tlatov, A.G., Guseva, S.A. \& Kim, G.D. 2001, in: V.I. Makarov, V. N. Obridko, (eds.), 'The Sun in Epoch of Magnetic Field Reversal', Proc of V Pulkovo International Conference on Solar Physics, St. Petersburg, Russia. 5, 385

Waldmeier, M. 1964, Zs. f. Astrophys. 59, 205 